\title{
Elucidating General Equilibrium Multiplier Effects: A Differential Perspective
}

\author{
M. Alejandro Cardenete ${ }^{1}$, Ferran Sancho ${ }^{2}$ \\ ${ }^{1}$ Department of Economics, Universidad Loyola Andalucía, Sevilla, Spain \\ ${ }^{2}$ Department of Economics, Universitat Autònoma de Barcelona, Barcelona, Spain \\ Email: macardenete@uloyola.es, ferran.sancho@uab.cat
}

Received August 20, 2013; revised September 11, 2013; accepted September 18, 2013

Copyright (C) 2013 M. Alejandro Cardenete, Ferran Sancho. This is an open access article distributed under the Creative Commons Attribution License, which permits unrestricted use, distribution, and reproduction in any medium, provided the original work is properly cited.

\begin{abstract}
Expenditure multipliers are routinely used to evaluate the effectiveness of government spending. When interested in disaggregated effects, interindustry models provide the necessary tools to be able to look at very detailed sectorial results. These models are theoretically simple and empirically operational, which makes them easily implementable and therefore popular with policy makers. They miss, however, quite a bit of the interaction that takes place at the micro level. On the one hand, they ignore the role exerted by supply constraints in primary factors; on the other hand, they look at the world as though it is fully linear. We overcome these limitations by using an opposite Walrasian general equilibrium model to compute marginal multipliers. By using differential calculus, we also offer some insights regarding the "under-the-hood" circuits of influence.
\end{abstract}

Keywords: Marginal Multipliers; General Equilibrium; Linear vs. Nonlinear Models

\section{Introduction}

Let us begin by considering the general setup of an economy described by $m$ endogenous variables and $k$ external exogenous variables, (say, policy instruments) affecting the equilibrium state. In this economy, multipliers connect exogenous injections $x_{i}(I=1,2 \cdots k)$ with endogenous responses $e_{j}(j=1,2 \cdots m)$. If the vector function $F: R^{m+k} \rightarrow R^{m}$ represents the equilibrium state $e=F(e, x)$, then it is possible to use differential calculus [1] to study the equilibrium dependence of endogenous variables $e$ with respect to exogenous one $x$. In this case, we would have

$$
d e=(\partial F(e, x) / \partial e) d e+(\partial F(e, x) / \partial X) d x .
$$

Solving now for de would yield

$$
\begin{aligned}
d e & =(\mathbf{I}-(\partial F(e, x) / \partial e))^{-1}(\partial F(e, x) / \partial x) d x \\
& =\mathbf{M}(e, x) d x
\end{aligned}
$$

where $\mathbf{M}$ is a $(m \times k)$ matrix whose generic element $\partial e_{j} / \partial x_{i}=m_{j i}(e, x)$ is an estimate of the (marginal) multiplier effect exerted by injection $x_{i}$ upon endogenous variable $e_{j}$. Notice that in principle the multiplier matrix, $\mathbf{M}$ itself may be variable since it depends on the particu- lar equilibrium state $e$ induced by instruments $x$ and the characteristics of the economy, as embodied in $F$. Since the vector function $F$ is not usually directly observable, neither are its derivatives nor is matrix $\mathbf{M}$, hence there is the need for relying on some type of approximation. One such approximation is to linearize the economy; another is to use numerical methods for the evaluation of nonlinear equilibrium relationships.

\section{Linear Multipliers}

We now consider a linear economy of the interindustry type. For this type of economies we have $n$ endogenous $(m=n)$ and $n$ exogenous variables $(k=n)$. The endogenous variables correspond to total output in each of the $n$ sectors, whereas the exogenous variables describe final demand. This includes discretional government demand for the goods and services of each of the $n$ sectors. We will use the $n$-vector $q$ to denote output (i.e. $e=q$ now) and keep $x$ for the exogenous variables. The equilibrium state for this linear economy is represented, again using the vector function $F$, by $q=F(q, x)$. Thanks to the linearity assumption this can be seen to adopt the form $q=$ $\mathbf{A} q+x$, where $\mathbf{A}$ is a $n \times n$ nonnegative, productive and homothetic technical coefficient matrix. From the equi- 
librium state we can quickly write the reduced form linking output with instruments as $q=(\mathbf{I}-\mathbf{A})^{-1} x=\mathbf{M} x$, with $\mathbf{M}$ representing the multiplier matrix of the linear economy. Because of the assumptions on $\mathbf{A}$, the matrix $\mathbf{M}$ is constant. Its entries are independent of the equilibrium state. Taking derivatives, it is quite simple now to relate changes in output with external changes in instruments

$$
\Delta q=(\mathbf{I}-\mathbf{A})^{-1} \Delta x=\mathbf{M} \Delta x
$$

Multipliers are given directly by the cells in matrix $\mathbf{M}$, i.e. $\partial q_{j} / \partial x_{i}=m_{j i}$. All that is needed to compute (linear) multipliers is the coefficients matrix A. Since this matrix is readily available from official statistics, this explains the popularity of linear models in policy oriented empirical economics. Even more, linear models are so simple that we need not worry about prices. Prices, in fact, can be seen to be completely independent from quantities in linear interindustry models. Notice that if quantities are not affected by prices, neither are multipliers. End of the story, all needed multiplier information is contained in matrix M. But we know that the actual story is bit more complicated than that since, in general, prices and quantities are mutually dependent.

\section{Applied General Equilibrium Multipliers}

In a standard general equilibrium model the interactions of supply and demand determine, at the same time, prices and quantities. We use now a general equilibrium framework to elucidate multipliers and compare them to their linear counterpart. Endogenous variables include now $n$ output levels $q$ and $n$ prices $p$, that is, $e=(q, p)$, so in total we have $2 n$ endogenous variables. Let us consider again that the government decides how much to buy of each of the $n$ goods and services; the government's demand levels are denoted by the vector $x$ representing policy instruments. The structural function $F$ representing the equilibrium state would now be of the type $F: R^{2 n+n} \rightarrow R^{2 n}$ which in turn can be split in two functions $F^{q}: R^{2 n+n} \rightarrow R^{n}$ and $F^{p}: R^{2 n+n} \rightarrow R^{n}$ determining quantities and prices, respectively. The complete general equilibrium state is represented by $(q, p)=F(q, p$, $x$ ), or using the fact that $F=F^{q} \times F^{p}$, it can also be seen as

$$
\begin{aligned}
& p=F^{p}(q, p, x) \\
& q=F^{q}(q, p, x)
\end{aligned}
$$

We perform comparative statics on the equilibrium state represented in Equation (2) considering an exogenous change $d x$ in the instruments $x$. We would obtain

$$
\begin{aligned}
& d q=M_{q q} d q+M_{q p} d p+M_{q x} d x \\
& d p=M_{p q} d q+M_{p p} d p+M_{p x} d x
\end{aligned}
$$

where we use, in Equation (3), the notational convention $M_{q q}=\partial F^{q}(q, p, x) / \partial q, M_{q p}=\partial F^{q}(q, p, x) / \partial p$, and so on. Solving for $d p$ in the second expression in Equation (3) and substituting the result in the first equation would yield

$$
\begin{aligned}
d q= & M_{q q} d q+M_{q p} d p+M_{q x} d x \\
= & M_{q q} d q+M_{q p}\left(I-M_{p p}\right)^{-1}\left(M_{p q} d q+M_{p x} d x\right) \\
& +M_{q x} d x \\
& =\left(M_{q q}+M_{q p}\left(I-M_{p p}\right)^{-1} M_{p q}\right) d q \\
& +\left(M_{q x}+M_{q p}\left(I-M_{p p}\right)^{-1} M_{p x}\right) d x
\end{aligned}
$$

Solving now for $d q$ in Equation (4) we finally obtain

$$
\begin{aligned}
& d q=\left(I-\left(M_{q q}+M_{q p}\left(I-M_{p p}\right)^{-1} M_{p q}\right)\right)^{-1} \\
& \cdot\left(M_{q x}+M_{q p}\left(I-M_{p p}\right)^{-1} M_{p x}\right)=\mathbf{M}(q, p, x) d x
\end{aligned}
$$

where $\mathbf{M}(q, p, x)$ stands for the general quantity multiplier matrix ${ }^{1}$. We now proceed to relate the linear multiplier matrix $\mathbf{M}$ in Equation (1) with the general multiplier matrix $\mathbf{M}(q, p, x)$ derived in Equation (5).

Recall first that in linear models quantities and prices are independently determined. Under this assumption the partial derivative matrices $M_{p q}$ and $M_{q p}$ would be such that $M_{p q}=M_{q p}=\mathbf{0}$ and then Equation (5) can be easily verified to reduce to

$$
d q=\left(I-M_{q q}\right)^{-1} M_{q x} d x
$$

The simplified expression that appears in Equation (6) is of course the differential version of the classical linear multiplier expression picked up in Equation (1) above, with $M_{q q}=\mathbf{A}$ and $M_{q x} d x=\Delta x$. The chains of interactions, however, can be seen to be quite more complex in Equation (5) than in Equation (1), in accordance with the higher complexity of nonlinear models vis-a-vis linear ones.

Figure 1 below depicts the way the model's interconnections work. Facing an external disturbance in $x$, the system first reacts with changes in prices and quantities through matrices $M_{p x}$ and $M_{q x}$. Price effects repeatedly self multiply through the loop $M_{p p}$ along the cost structure which, in turn, are affected by cross effects $M_{q p}$ from quantities to prices. Similarly, the initial effect of the disturbance on quantities gets itself multiplied by the chain reaction that moves directly from quantities to quantities, i.e. $M_{q q}$, and indirectly from quantities to looped prices and back to quantities via the combined

\footnotetext{
${ }^{1} \mathrm{~A}$ similar derivation, that we omit, would produce a general price multiplier matrix.
} 


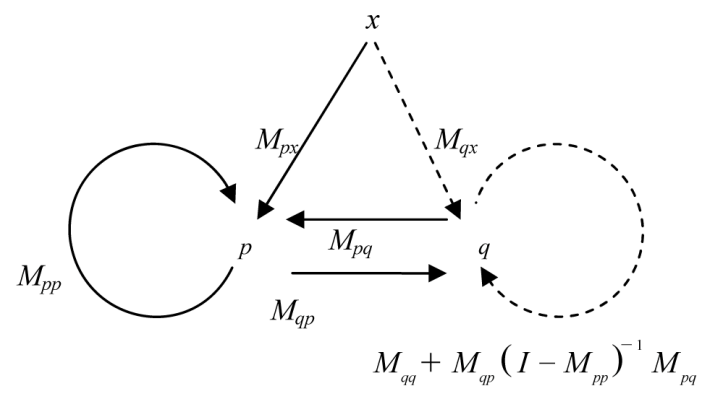

Figure 1. Example of the circuits of influence.

effect measured by

$$
M_{q p}\left(I-M_{p p}\right)^{-1} M_{q p} .
$$

Notice again the role exerted by cross effects, in this case from prices to quantities through $M_{q p}$. The dashed arrows show the only influence that would remain in the typical linear model where prices and quantities are independently determined. The linear model effects would be restricted to matrices $M_{q x}$ and $M_{q q}$.

\section{Some Numerical Results}

We show now some results of implementing these two models, namely, a linear interindustry model first (see [2] for a reference of linear models), and then a Walrasian general equilibrium model (see $[3,4]$ for examples and details of empirical general equilibrium models and their methodology). We use data from the Spanish economy [5] for 2006 to calibrate both models. Calibration entails the selection of parameters so as to reproduce the empirical data as an equilibrium under both models - the linear and Walrasian versions (see [6] for a step-by-step guide to calibration). The Spanish data distinguishes 26 productive sectors. For each sector we show the multiplier effects under the two models in Table 1 below. For instance, the linear multiplier value of 1.4620 indicates the (positive) change in economy-wide production when a unitary exogenous demand for Agriculture is injected into the economy. The general equilibrium multiplier of -0.5341 tells a different story. Now overall production would go down, once all general equilibrium adjustments had taken place. Supply restrictions and interconnected price and quantity effects explain the different sign. The initial injection into Agriculture is not able to activate any overall output increase. The need for more primary factors to satisfy the extra demand for Agriculture requires siphoning them from elsewhere in the economy, triggering an economy-wide fall in production. The indirect output substitution effects more than compensate the direct output volume effect arising from the extra injection.

Unlike the general positive multiplier effects of linear models, multipliers results can perfectly be negative in a
Table 1. Multiplier comparison.

\begin{tabular}{|c|c|c|c|}
\hline \multirow{2}{*}{$\begin{array}{c}\text { Spanish } \\
\text { Data }\end{array}$} & \multicolumn{3}{|l|}{ Multiplier Estimates } \\
\hline & Economic Sector & $\begin{array}{c}\text { Linear } \\
\text { Multipliers }\end{array}$ & $\begin{array}{l}\text { Gen. Equilib. } \\
\text { Multipliers }\end{array}$ \\
\hline 1. & Agriculture & 1.4620 & -0.5431 \\
\hline 2. & Fisheries & 1.0394 & 0.3305 \\
\hline 3. & Coal & 1.0211 & 0.5179 \\
\hline 4. & Petroleum and Gas & 1.0056 & 0.9825 \\
\hline 5. & Mining & 1.0974 & 0.3106 \\
\hline 6. & Oil Refining & 2.0322 & 1.3076 \\
\hline 7. & Electricity & 3.1015 & 0.2129 \\
\hline 8. & Gas & 1.2480 & 0.8665 \\
\hline 9. & Water & 1.0640 & -0.5126 \\
\hline 10. & Foodstuffs & 2.7998 & 0.4838 \\
\hline 11. & Clothing & 1.3781 & 0.7127 \\
\hline 12. & Wood Products & 1.5952 & 0.2936 \\
\hline 13. & Chemicals & 1.8607 & 0.6111 \\
\hline 14. & Building Materials & 2.0411 & 0.0953 \\
\hline 15. & Iron and Steel & 2.2508 & 0.5351 \\
\hline 16. & Metal Products & 2.0267 & 0.2699 \\
\hline 17. & Machinery & 2.1037 & 0.6224 \\
\hline 18. & Vehicles & 2.1284 & 0.8703 \\
\hline 19. & Other Transport. Equip. & 1.3384 & 0.5763 \\
\hline 20. & Other Manufactures & 2.2511 & 0.2646 \\
\hline 21. & Construction & 6.2904 & -0.0082 \\
\hline 22. & Commerce & 3.5528 & -1.7232 \\
\hline 23. & Transport. and Comm. & 2.8001 & -03582 \\
\hline 24. & Other Services & 2.2807 & -0.6793 \\
\hline 25. & Services for Sale & 2.2151 & -1.1277 \\
\hline \multirow[t]{2}{*}{26.} & Public Services & 2.2962 & $-1-1607$ \\
\hline & Total multiplier effect & 54.2805 & 3.7503 \\
\hline
\end{tabular}

Walrasian general equilibrium model. Moreover, the combined multiplier effects can be dramatically different in size. Under the linear model, aggregate output effects can be as high as 54.2805 , meaning that an additional unitary demand for each and every of the 26 sectors would give rise, on average, to a multiplier effect of $54.2805 / 26=2.0877$ new output units. The general equilibrium model, however, reduces this estimate drastically, with an average value of only $3.7503 / 26=0.1442$ new units of output. 


\section{Conclusion}

The interaction of demand and supply in goods, services and productive factors in response to external, policy oriented induced changes makes multiplier estimates be substantially smaller, and even negatively valued, in a general equilibrium model than in a linear model where price effects are disregarded and resource constraints are not binding. Expenditure policies designed upon optimistically estimated linear multiplier values should therefore be carefully reevaluated, and perhaps even abandoned. In fact the use of the name "multiplier" could even be a misnomer. Under general equilibrium, multipliers are not systematically above 1 , or even positive in sign for that matter; hence output levels need not "multiply" over 1 , as the standard linear models conclude. The tradition is however too strong to be changed, and we will still refer to the effects of external injections in endogenous output as "multipliers", provided the modeling assumptions under which they are estimated are explicitly laid out.

\section{Acknowledgements}

Support from research projects MINECO2009-11857 and SGR2009-587 is gratefully acknowledged. The usual caveat applies.

\section{REFERENCES}

[1] G. Debreu, "Economies with a Finite Set of Equilibria," Econometrica, Vol. 38, No. 3, 1970, pp. 387-392. http://dx.doi.org/10.2307/1909545

[2] R. E. Miller and P. D. Blair, "Input-Output Analysis: Foundations and Extensions," Cambridge University Press, Cambridge, 2009. http://dx.doi.org/10.1017/CBO9780511626982

[3] J. Shoven and J. Whalley, "Applied General Equilibrium Models of Taxation and International Trade," Journal of Economic Literature, Vol. 22, No. 3, 1984, pp. 1007 1051.

[4] T. J. Kehoe, T. N. Srinivisan and J. Whalley, "Frontiers in Applied General Equilibrium Modeling," Oxford University Press, Oxford, 2005. http://dx.doi.org/10.1017/CBO9780511614330

[5] J. M. Cansino, M. A. Cardenete, M. Ordóñez and R. Román, "Análisis de Los Sectores Clave de la Economía Española a Partir de la Matriz de Contabilidad Social de España 2006," University of Seville, Unpublished Mimeo, 2011.

[6] F. Sancho, "Calibration of CES Functions for 'Realworld' Multisectoral Modeling," Economic Systems Research, Vol. 21, No. 1, 2009, pp. 45-58. http://dx.doi.org/10.1080/09535310902731270 